DSF-20-02

hep-th/0209174

September2002

\title{
Generalized Weyl Systems and $\kappa$-Minkowski space
}

\author{
Alessandra Agostini, Fedele Lizzi and Alessandro Zampini \\ Dipartimento di Scienze Fisiche, Università di Napoli Federico II \\ and \\ INFN, Sezione di Napoli \\ Monte S. Angelo, Via Cintia, 80126 Napoli, Italy \\ alessandra.agostini, fedele.lizzi, alessandro.zampini @na.infn.it
}

\begin{abstract}
We introduce the notion of generalized Weyl system, and use it to define *products which generalize the commutation relations of Lie algebras. In particular we study in a comparative way various $*$-products which generalize the $\kappa$-Minkowski commutation relation.
\end{abstract}




\section{Introduction}

The recent nearly explosive interest in Noncommutative Geometry [1], 2] has been mainly concentrated on a noncommutative space on which the coordinates have the canonical structure [3, 4], their commutator being a constant:

$$
\left[x_{\mu}, x_{\nu}\right]=-i \theta_{\mu \nu}
$$

This is of course just an example of a noncommutative space, albeit a very important one for its connections with quantum mechanics. In general, one of the most fruitful ways to deal with such spaces is via the definition of a deformation of the usual product among functions on the space. This way the noncommutative space is studied as the structure space of a deformed $*$-algebra円. The definition of this $*$-algebra is very important, as it is the first step towards the use of Connes' machinery for the construction of physical theories, and the construction of field theories on noncommutative spaces. In the deformed algebra, functions are not multiplied with the commutative (pointwise) product, but with a new, noncommutative, product. For example equation (1.1) becomes

$$
x_{\mu} \star x_{\nu}-x_{\nu} \star x_{\mu}=-i \theta_{\mu \nu}
$$

One of the ways to reproduce this relation is via the introduction of the Moyal product [5]:

$$
(f \star g)(x):=\frac{1}{\left(\pi^{2 n}\right) \operatorname{det}(\theta)} \int_{\mathbb{R}^{2 n}} \int_{\mathbb{R}^{2 n}} d^{2 n} y d^{2 n} z e^{2 i\left(x^{t} \theta^{-1} y+y^{t} \theta^{-1} z+z^{t} \theta^{-1} x\right)} f(y) g(z)
$$

This product is probably more familiar with the asymptotic expansion

$$
\left.(f \star g)(x) \equiv e^{-\frac{i}{2} \theta_{\mu \nu} \partial^{y \mu} \partial^{z_{\nu}}} f(y) g(z)\right|_{y=z=x}
$$

which however is valid on a smaller domain than (1.3). In terms of this product the exponentiated version of (1.1) reads:

$$
e^{i k^{\mu} x_{\mu}} \star e^{i l^{\nu} x_{\nu}}=e^{\frac{i}{2} k^{\mu} \theta_{\mu \nu} l^{\nu}} e^{i(k+l)^{\mu} x_{\mu}}
$$

Generalized deformed *-products were originally introduced in [6] as a first attempt to develop a quantization of classical dynamics on phase space. In fact, such a deformed product can be defined on a vector space equipped with a constant symplectic structure. A slightly more general case is the introduction of a deformed product on a vector space on which a constant, at first, Poisson bracket is defined. It has the property that the $*$-commutator of two functions reduces to the Poisson bracket to first order in the deformation parameter:

$$
[f, g]_{*}=-i \theta\{f, g\}+O\left(\theta^{3}\right)
$$

\footnotetext{
${ }^{1}$ The $*$ here refers to the presence of an hermitean (complex) conjugation, and has nothing to do with the deformed products we will introduce later on.
} 
A manifold on which a Poisson bracket has been defined is called a Poisson Manifold. All symplectic manifolds, on which a nonsingular two-form $\omega$ is defined, are naturally Poisson manifolds, defined in terms of the bivector $\Lambda$, inverse of $-\omega$ :

$$
\{f, g\}=\Lambda_{\mu \nu} \partial^{\mu} f \partial^{\nu} g
$$

Not all Poisson manifolds are however symplectic. The general problem of finding a deformed product for a general Poisson manifold has been solved by Kontsevich [7], at least at the level of formal series, that is, without considerations of the convergence of the series.

A noncommutative space is $\kappa$-Minkowski, defined by:

$$
\begin{aligned}
{\left[x_{0}, x_{i}\right] } & =\frac{i}{\kappa} x_{i}=i \lambda x_{i} \\
{\left[x_{i}, x_{j}\right] } & =0
\end{aligned}
$$

The importance of $\kappa$-Minkowski (and the origin of the namef) lies in the fact that it is the homogeneous space for the quantum deformation of the $D=4$ Poincaré algebra called $U_{\kappa}\left(\mathcal{P}_{4}\right)$ [8]. This deformation of the Poincaré algebra has been originally presented in [9] by contraction? of a real form of the quantum anti-de Sitter algebra $U_{q}(s o(3,2))$ with a procedure introduced by [11]. The choice of the generators of $U_{\kappa}\left(\mathcal{P}_{4}\right)$ is not unique, different basic generators modify its form. Expressing its generators in the bicrossproduct basis [12, it is possible to see that $\kappa$-Poincaré acts covariantly as an Hopf algebra on a noncommutative space whose generators obey equation (1.8). In the limit $\kappa \rightarrow \infty$ $(\lambda \rightarrow 0)$ one recovers the standard Minkowski space, with the ordinary Poincaré group. $\kappa$-Minkowski is naturally a Poisson manifold, with bracket:

$$
\{f, g\}=x_{i}\left(\partial^{i} f \partial^{0} g-\partial^{0} f \partial^{i} g\right)
$$

A crucial aspect of these relations is that they define a solvable Lie algebra on the generators, and this makes them particular cases of more general products.

The aim of this paper is to compare different products present in the literature, which satisfy (1.8) and show that they can be seen as generalizations of the Moyal product, or rather of the exponentiated version of eq. (1.5), based on a more general notion of Weyl system. Along the way we will develop a method for the construction of deformed products, that enable to obtain a wider class of commutation relations among the coordinate functions, reproducing Lie algebra structure with the possibility of considering central extension as well.

The paper is organized as follows. In section 2 we introduce the main tool of our investigation: Weyl systems, and generalize them. In section 3 we review a number of deformed $\kappa$-Minkowski products, and in section 4 we show that they can all be written in terms of generalized Weyl systems. Some conclusions follow. Technical details of the calculations are in the appendix.

\footnotetext{
${ }^{2}$ The notation with the parameter $\lambda=1 / \kappa$ is also common, and we prefer it in this paper.

${ }^{3}$ For an alternative construction see, for example 10$]$.
} 


\section{Weyl System and maps and their Generalization}

\subsection{Standard Weyl Systems and Maps}

The concept of what can now be referred to as a standard Weyl system was introduced by H. Weyl [13]. The main motivation at the time was to avoid the presence of unbounded operators in the quantum mechanical formalism. We will use the concept (and its generalizations presented below) as a tool to construct $*$-algebras whose generators satisfy some commutation relations.

We start with a brief description of the standard Weyl systems [14. Given a real, finite dimensional, symplectic vector space $S$, a Weyl system is a map between this space and the set of unitary operators on a suitable Hilbert space:

$$
W: S \mapsto \mathcal{U}(\mathcal{H})
$$

with the property:

$$
W\left(k+k^{\prime}\right)=e^{-\frac{i}{2} \omega\left(k, k^{\prime}\right)} W(k) W\left(k^{\prime}\right)
$$

where $\omega$ is the symplectic, translationally invariant, form on $S$. On each one-dimensional subspace of $S$, this formula reduces to ( $\alpha$ and $\beta$ real scalars):

$$
W((\alpha+\beta) k)=W(\alpha k) W(\beta k)=W(\beta k) W(\alpha k)
$$

This means that, for each $k, W(\alpha k)$ is a one parameter group of unitary operators. According to Stone's theorem $W$ is the exponential of a hermitian operator on $\mathcal{H}$ :

$$
W(\alpha k)=e^{i \alpha X(k)}
$$

and the vector space structure implies that

$$
X(\alpha k)=\alpha X(k)
$$

Relation (2.2) can be cast in the form

$$
W(k) W\left(k^{\prime}\right)=e^{i \omega\left(k, k^{\prime}\right)} W\left(k^{\prime}\right) W(k)
$$

This can be considered the exponentiated version of the commutation relations, thus satisfying the original Weyl motivation. The usual form of the commutation relations between generators can be recovered with a series expansion

$$
\left[X(k), X\left(k^{\prime}\right)\right]=-i \omega\left(k, k^{\prime}\right)
$$

In the usual identification of $S$ with $\mathbb{R}^{2 n}$, the cotangent bundle of $\mathbb{R}^{n}$, with canonical coordinates $\left(q^{i}, p_{j}\right)$ and $\omega=d q^{j} \wedge d p_{j}$, this construction can be given an explicit realization

$$
W(q, p)=e^{i\left(q^{j} P_{j}+p_{j} Q^{j}\right)}
$$


where $P_{j}$ and $Q^{j}$ are the usual operators that represents the position and momentum observables for a system of particles, whose dynamics is classically described on the phase space $\mathbb{R}^{2 n}$. This form of the operator $W$ suggests how to relate an operator on $\mathcal{H}$ to a function defined on $S$. It reminds the integral kernel used to define the Fourier transform. It can be intuitively seen as a sort of "plane wave basis" in a set of operatorst. Given a function $f$ on the phase space, whose coordinates we collectively indicate with $x$, with Fourier transform

$$
\tilde{f}(k)=\frac{1}{(2 \pi)^{n}} \int d^{2 n} x f(x) e^{-i k x}
$$

we define the operator $\Omega(f)$ via the Weyl map

$$
\Omega(f)=\frac{1}{(2 \pi)^{n}} \int d^{2 n} k \tilde{f}(k) W(k)
$$

Where by $k x$ we mean $k^{\mu} x_{\mu}$. $\Omega(f)$ is the operator that, in the $W(k)$ basis, has coefficients given by the Fourier transform of $f$. The inverse of the Weyl map, also called the Wigner map, maps an operator $F$ into a function, whose Fourier transform is:

$$
\Omega^{-1}(F)(k)=\operatorname{Tr} F W^{\dagger}(k)
$$

The bijection $\Omega$ can now be used to translate the composition law in the set of operators on $\mathcal{H}$ into an associative, non abelian, composition law in the space of function defined on $\mathbb{R}^{2 n}$. For two functions on $\mathbb{R}^{2 n}$ we define the $\star$ product as

$$
(f \star g)=\Omega^{-1}(\Omega(f) \Omega(g))
$$

For a Weyl system defined by (2.2), with $\omega$ such that $\omega\left(k, k^{\prime}\right)=k^{\mu} \theta_{\mu \nu} k^{\prime \nu}$, this reduces to the product defined in (1.3) or (1.4). If we consider to perform Fourier transform in a distributional sense, then it is possible to define Moyal product between coordinate functions, thus obtaining

$$
x_{\mu} \star x_{\nu}=x_{\mu} x_{\nu}-\frac{i}{2} \theta_{\mu \nu}
$$

and relation (1.1).

The deformed algebra just defined is a *-algebraf with norm

$$
\|f\|=\sup _{g \neq 0} \frac{\|f \star g\|_{2}}{\|g\|_{2}}
$$

with $\|\cdot\|_{2}$ the $L^{2}$ norm defined as

$$
\left(\|f\|_{2}\right)^{2} \equiv \int d^{2 n} k|\tilde{f}(k)|^{2}
$$

\footnotetext{
${ }^{4}$ Our considerations are valid for rapid descent Schwarzian functions, and in the following we will not pay particular attention to the domain of definition of the product, discussed at length in [15].

${ }^{5}$ We leave aside issues of norm completeness of the algebra.
} 
The hermitean conjugation is the usual complex conjugation. Note that it results

$$
\Omega\left(f^{*}\right)=\Omega(f)^{\dagger}
$$

These two ingredients enable to give this set of functions a very important structure in the context of non commutative geometry formalism. It is possible to see that Weyl map $\Omega$ is an example of the GNS construction (see [2]) which represents any $C^{*}$-algebra as bounded operators on a Hilbert space.

\subsection{Generalized Weyl Systems}

In the last section we have shown how the Moyal product arises via an explicit realization of the Weyl system, in terms of unitary operators on a Hilbert space, and how it is nothing but a realization, in the space of functions, of the operator composition law. Now we show how it is possible to define a class of "deformed" products in a set of function defined on $\mathbb{R}^{n}$, without using an explicit realization of a Weyl system, thus opening the possibility for a generalization of this concept.

While we have previously considered a standard Weyl system simply as a map, now we want to consider it as a unitary projective representation of the translations group in an even dimensional real vector space. The most natural generalization is to consider the manifold $\mathbb{R}^{n}$, and $\oplus$, a non-abelian composition law between points. Thus $\mathbb{R}^{n}$ acquires a general Lie group structure. A generalized Weyl system is the map in the set of operators $W(k)$ with the composition rule

$$
W(k) W\left(k^{\prime}\right)=e^{\frac{i}{2} \omega\left(k, k^{\prime}\right)} W\left(k \oplus k^{\prime}\right)
$$

For the algebra to be associative it must be

$$
\omega\left(k^{1}, k^{2} \oplus k^{3}\right)+\omega\left(k^{2}, k^{3}\right)=\omega\left(k^{1}, k^{2}\right)+\omega\left(k^{1} \oplus k^{2}, k^{3}\right)
$$

Without entering into a cohomological characterization of this relation, it is enough to mention that such a $\omega$ is called a cocycle. If $\omega\left(k, k^{\prime}\right)$ is bilinear in both its entries, then it is necessary a cocycle. In the Moyal case $\omega$ is two-form and therefore bilinear in both entries.

Since we are looking for deformations of the algebra driven by a parameter $\lambda$, we also require that

$$
\begin{aligned}
\lim _{\lambda \rightarrow 0} k \oplus k^{\prime} & =k+k^{\prime} \\
\lim _{\lambda \rightarrow 0} \omega\left(k, k^{\prime}\right) & =0
\end{aligned}
$$

In analogy with the Moyal case we define a map $\Omega$ from ordinary functions to formal elements of a noncommutative algebra as

$$
\Omega(f) \equiv F \equiv \frac{1}{(2 \pi)^{n / 2}} \int d^{n} k \tilde{f}(k) W(k)
$$

\footnotetext{
${ }^{6}$ We will denote the elements of the deformed algebra with capital letters.
} 
where the product is

$$
\Omega(f) \Omega(g)=F G=\frac{1}{(2 \pi)^{n}} \int d^{n} k d^{n} k^{\prime} \tilde{f}(k) \tilde{g}\left(k^{\prime}\right) W(k) W\left(k^{\prime}\right)
$$

Formally using the definition of generalized Weyl systems we obtain

$$
F G=\frac{1}{(2 \pi)^{n}} \int d^{n} k d^{n} k^{\prime} \tilde{f}(k) \tilde{g}\left(k^{\prime}\right) e^{\frac{i}{2} \omega\left(k, k^{\prime}\right)} W\left(k \oplus k^{\prime}\right)
$$

It is useful to write the product as a twisted convolution. In order to do this, let us define the inverse of $k$ with respect to the composition law $\oplus$ ):

$$
\bar{k}: k \oplus \bar{k}=\bar{k} \oplus k=0
$$

Note that we are assuming that the right and the left inverse are the same. With a change of variables in eq. (2.22)

$$
k \oplus k^{\prime}=\xi
$$

then

$$
k=\xi \oplus \bar{k}^{\prime}=\alpha\left(\xi, \bar{k}^{\prime}\right)
$$

Equation (2.22) becomes

$$
\begin{aligned}
F G & =\frac{1}{(2 \pi)^{n}} \int d^{n} k^{\prime} d^{n} \xi J\left(\xi, k^{\prime}\right) \tilde{f}\left(\xi \oplus \overline{k^{\prime}}\right) \tilde{g}\left(k^{\prime}\right) e^{\frac{i}{2} \omega\left(\xi \oplus \bar{k}^{\prime}, k^{\prime}\right)} W(\xi) \\
& =\frac{1}{(2 \pi)^{n}} \int d^{n} \xi d^{n} k^{\prime} J\left(\xi, k^{\prime}\right) \tilde{f}\left(\alpha\left(\xi, \bar{k}^{\prime}\right)\right) \tilde{g}\left(k^{\prime}\right) e^{\frac{i}{2} \omega\left(\alpha, k^{\prime}\right)} W(\xi)
\end{aligned}
$$

where $J\left(\xi, k^{\prime}\right)=\left|\partial_{\xi} \alpha\left(\xi, \bar{k}^{\prime}\right)\right|$ is the Jacobian of the transformation (2.25). The last equation can be cast in a more suggestive form

$$
F G=\frac{1}{(2 \pi)^{n / 2}} \int d^{n} \xi\left(\frac{1}{(2 \pi)^{n / 2}} \int d^{n} k^{\prime} J\left(\xi, k^{\prime}\right) \tilde{f}\left(\alpha\left(\xi, \bar{k}^{\prime}\right)\right) \tilde{g}\left(k^{\prime}\right) e^{\frac{i}{2} \omega\left(\alpha, k^{\prime}\right)}\right) W(\xi)
$$

Comparison with (2.20) suggests to define the Fourier transform of the deformed product of $f$ and $g$ as:

$$
\widetilde{(f * g)}(\xi)=\frac{1}{(2 \pi)^{n / 2}} \int d^{n} k^{\prime} J\left(\xi, k^{\prime}\right) \tilde{f}\left(\alpha\left(\xi, \overline{k^{\prime}}\right)\right) \tilde{g}\left(k^{\prime}\right) e^{\frac{i}{2} \omega\left(\alpha, k^{\prime}\right)}
$$

A detailed calculation shows that

$$
(f * g)(x)=\frac{1}{(2 \pi)^{n}} \int d^{n} k d^{n} k^{\prime} \tilde{f}(k) \tilde{g}\left(k^{\prime}\right) e^{\frac{i}{2} \omega\left(k, k^{\prime}\right)} e^{i\left(k \oplus k^{\prime}\right) x}
$$

This definition enables to write $\Omega\left(e^{i k x}\right)=W(k)$, and

$$
\Omega^{-1}(W(k))=e^{i k x}
$$

\footnotetext{
${ }^{7}$ Here 0 denotes the neutral element of $\left(\mathbb{R}^{n}, \oplus\right)$.
} 
which gives the inverse of the map (2.20) for all operators that can be "expanded" in the "plane wave" basis given by the $W$ 's. As we claimed, it has been possible to define a product simply considering $W$ as a formal device. Now we have given the set of function on $\mathbb{R}^{n}$ a structure of algebra. It is easy to check that the neutral element of the product is $W(0)$, which, as is usual for noncompact geometries, does not belong to the algebra, which is composed of functions which vanish at infinity; while associativity is a consequence of the associativity of $\oplus$.

To define an hermitean conjugation, we use the fact that for the undeformed algebra

$$
f(x)^{\dagger}=f(x)^{*}=\frac{1}{(2 \pi)^{n / 2}} \int d^{n} k \tilde{f}^{*}(k) e^{-i k x}=\frac{1}{(2 \pi)^{n / 2}} \int d^{n} k \tilde{f}^{*}(-k) e^{i k x}
$$

and define the hermitean conjugate of $F$, defined as in eq. (2.20) as

$$
F^{\dagger}=\frac{1}{(2 \pi)^{n / 2}} \int d^{n} k \tilde{f}^{*}(\bar{k}) W(k)
$$

This means that we assume, in the set of functions

$$
f^{\dagger}(x)=\frac{1}{(2 \pi)^{n}} \int d^{n} k d^{n} a f^{*}(a) e^{i k x} e^{i \bar{k} a}
$$

The norm is defined as in (2.14). The compatibility of the hermitean conjugation with the product (2.22):

$$
(F G)^{\dagger}=G^{\dagger} F^{\dagger}
$$

imposes further restrictions on $\omega$ and $\oplus$. Using the definition of hermitean conjugate we obtain:

$$
(F G)^{\dagger}=\left.\frac{1}{(2 \pi)^{n}} \int d^{n} \xi d^{n} k^{\prime} J^{*}\left(\xi^{\prime}, k^{\prime}\right)\right|_{\xi^{\prime}=\bar{\xi}} \tilde{f}^{*}\left(\alpha\left(\bar{\xi}, \bar{k}^{\prime}\right)\right) \tilde{g}^{*}\left(k^{\prime}\right) e^{-\frac{i}{2} \omega^{*}\left(\alpha\left(\bar{\xi}, \bar{k}^{\prime}\right), k^{\prime}\right)} W(\xi)
$$

on the other hand if we compute

$$
\begin{aligned}
G^{\dagger} F^{\dagger} & =\frac{1}{(2 \pi)^{n}} \int d^{n} k d^{n} p \tilde{g}^{*}(\bar{k}) \tilde{f}^{*}(\bar{p}) W(k) W(p) \\
& =\frac{1}{(2 \pi)^{n}} \int d^{n} k^{\prime} d^{n} \xi J^{\prime}\left(\xi, k^{\prime}\right) \tilde{g}^{*}\left(k^{\prime}\right) \tilde{f}^{*}\left(\overline{\left(k^{\prime} \oplus \xi\right)}\right) e^{\frac{i}{2} \omega\left(\overline{k^{\prime}}, k^{\prime} \oplus \xi\right)} W(\xi) \\
& =\frac{1}{(2 \pi)^{n}} \int d^{n} k^{\prime} d^{n} \xi J^{\prime}\left(\xi, k^{\prime}\right) \tilde{g}^{*}\left(k^{\prime}\right) \tilde{f}^{*}\left(\alpha\left(\bar{\xi}, \overline{k^{\prime}}\right)\right) e^{\frac{i}{2} \omega\left(\overline{k^{\prime}}, \alpha\left(k^{\prime}, \xi\right)\right)} W(\xi)
\end{aligned}
$$

where $k^{\prime}=\bar{k}$ and $k \oplus p=\xi$, then

$$
p=\bar{k} \oplus \xi=k^{\prime} \oplus \xi \Rightarrow \bar{p}=\bar{\xi} \oplus \bar{k}^{\prime}
$$

if and only if

$$
\overline{\left(k \oplus k^{\prime}\right)}=\overline{k^{\prime} \oplus \bar{k}}
$$


This is a further requirement on $\oplus$, that makes it into a group. The Jacobian becomes

$$
J^{\prime}\left(\xi, k^{\prime}\right)=\left|\partial_{k^{\prime}} \overline{k^{\prime}} \partial_{\xi} \alpha\left(k^{\prime}, \xi\right)\right|
$$

Comparing the two equations (2.35) and (2.36), we obtain sufficient conditions for compatibility

$$
\begin{aligned}
J^{\prime}\left(\xi, k^{\prime}\right) \equiv\left|\partial_{k^{\prime}} \bar{k}^{\prime} \partial_{\xi} \alpha\left(k^{\prime}, \xi\right)\right| & =\left.\left|\partial_{\xi^{\prime}} \alpha^{*}\left(\xi^{\prime}, \overline{k^{\prime}}\right)\right|_{\xi^{\prime}=\bar{\xi}} \equiv J^{*}\left(\xi^{\prime}, k^{\prime}\right)\right|_{\xi^{\prime}=\bar{\xi}} \\
\omega^{*}\left(\alpha\left(\bar{\xi}, \overline{k^{\prime}}\right), k^{\prime}\right) & =-\omega\left(\bar{k}^{\prime}, \alpha\left(k^{\prime}, \xi\right)\right)
\end{aligned}
$$

The standard Weyl-Moyal system described in the previous section is an example of this construction, with $\oplus$ the usual sum. Once an abstract $*$-algebra has been defined, it is in principle possible to construct an Hilbert space on which the algebra is represented by bounded operators. This could eventually be done with the GNS construction, enabling to recover $W$ as explicitly realized operators.

As we said, the $\oplus$ has given a group structure to "momentum" space, and of course there will be a Lie algebra associated to the group. We now will argue that this Lie algebra structure is the same of the noncommutativity of the $x$ 's on the deformed space. Define first of all the generators $x_{i}$ 's

$$
X_{\alpha}=\frac{1}{(2 \pi)^{n}} \int d^{n} x d^{n} k x_{\alpha} e^{-i k x} W(k)
$$

Product between them is (performing the integral in a distributional sense, with suitable boundary conditions)

$$
\begin{aligned}
x_{\alpha} * x_{\beta} & =\frac{1}{(2 \pi)^{2 n}} \int d^{n} z d^{n} y d^{n} k d^{n} l z_{\alpha} y_{\beta} e^{-i(k z+l y)} e^{\frac{i}{2} \omega(k, l)} e^{i x(k \oplus l)} \\
& =-\left.\frac{i}{2}\left(\frac{\partial^{2} \omega(k, l)}{\partial k_{\alpha} \partial l_{\beta}}\right)\right|_{k=l=0}-\left.i x_{\mu}\left(\frac{\partial^{2}}{\partial k_{\alpha} \partial l_{\beta}}(k \oplus l)^{\mu}\right)\right|_{k=l=0}+x_{\alpha} x_{\beta}
\end{aligned}
$$

The commutator is given by the antisymmetric combination of this product. It is possible to prove that the second term in the r.h.s. of this relation gives the structure constants of the Lie algebra defined by the Lie group $\left(\mathbb{R}^{n}, \oplus\right)$ :

$$
\left(\frac{\partial^{2}}{\partial k_{\alpha} \partial l_{\beta}}(k \oplus l)^{\mu}\right)-\left.\left(\frac{\partial^{2}}{\partial k_{\beta} \partial l_{\alpha}}(k \oplus l)^{\mu}\right)\right|_{k=l=0}=c_{\alpha \beta}^{\mu}
$$

while the cocycle term gives a central extension that can be cast in the usual form:

$$
\left.\frac{1}{2}\left(\frac{\partial^{2} \omega(k, l)}{\partial k_{\alpha} \partial l_{\beta}}-\frac{\partial^{2} \omega(k, l)}{\partial k_{\beta} \partial l_{\alpha}}\right)\right|_{k=l=0}=\theta_{\alpha \beta}
$$

Finally one obtains:

$$
\left[x_{\alpha}, x_{\beta}\right]_{*}=-i c_{\alpha \beta}^{\mu} x_{\mu}-i \theta_{\alpha \beta}
$$

In the following we will consider $\kappa$-Minkowski without central extensions, and therefore set $\theta=0$. 


\section{3 *-products on $\kappa$-Minkowski}

So far we have abstractly defined a generalization of a Weyl system. In this section we will show that these systems are a good description of some $*$-products in the study of the $\kappa$-Minkowski space which we briefly describe in this section. The noncommutative space $\kappa$-Minkowski is intimately related to the $\kappa$-deformed Poincaré algebra, $U_{\kappa}\left(\mathcal{P}_{4}\right)$. This is a quantum group originally obtained in [9], by contraction of the $q$-deformed anti-de Sitter algebra in the so-called standard basis. Subsequently in [12] a new basis was found (bicrossproduct basis) in which the Lorentz sector is not deformed, the deformation occurs only in the cross-relations between the Lorentz and translational sectors 8 :

$$
\begin{aligned}
{\left[P_{\mu}, P_{\nu}\right] } & =0 \\
{\left[M_{i}, P_{j}\right] } & =i \epsilon_{i j k} P_{k} \\
{\left[M_{i}, P_{0}\right] } & =0 \\
{\left[N_{i}, P_{j}\right] } & =-i \delta_{i j}\left(\frac{1}{2 \lambda}\left(1-e^{2 \lambda P_{0}}\right)+\frac{\lambda}{2} P^{2}\right)+i \lambda P_{i} P_{j} \\
{\left[N_{i}, P_{0}\right] } & =i P_{i}
\end{aligned}
$$

and the Lorentz subalgebra remains classical:

$$
\begin{aligned}
{\left[M_{i}, M_{j}\right] } & =i \epsilon_{i j k} M_{k} \\
{\left[M_{i}, N_{j}\right] } & =i \epsilon_{i j k} N_{k} \\
{\left[N_{i}, N_{j}\right] } & =-i \epsilon_{i j k} M_{k}
\end{aligned}
$$

All these commutation relations becomes the standard ones for $\lambda \rightarrow 0$. The bicrossproduct basis is peculiar as $\kappa$-Poincaré acts covariantly on a space that is necessarily deformed and noncommutative. This is a consequence of the non cocommutativity of the coproduct which, always in the bicrossproduct basis, reads:

$$
\begin{aligned}
\Delta P_{0} & =P_{0} \otimes 1+1 \otimes P_{0} \\
\Delta M_{i} & =M_{i} \otimes 1+1 \otimes M_{i} \\
\Delta P_{i} & =P_{i} \otimes 1+e^{\lambda P_{0}} \otimes P_{i} \\
\Delta N_{i} & =N_{i} \otimes 1+e^{+\lambda P_{0}} \otimes N_{i}+\lambda \varepsilon_{i j k} P_{j} \otimes M_{k}
\end{aligned}
$$

The quantum algebra (3.1) contains a translation subalgebra, and it is natural to consider the dual of the enveloping algebra of translations as $\kappa$-Minkowski space, on which $\kappa$ Poincaré acts covariantly. Because of the non cocommutative relations (3.3) the generators of the dual space do not commute, and their commutation relations is given by $(1.8)$. The possibility that at high energies the symmetry of quantum gravity may be of the quantum kind has created some interest in the study of $\kappa$-Minkowski spaces. Deformations of the

\footnotetext{
${ }^{8}$ We will usually use four-dimensional greek indices $(\mu, \nu=0, \ldots, 3)$ and three dimensional latin indices $(i, j=1,2,3)$, for $\kappa$-Minkowski we use a $(+,-,-,-)$ signature. Many of our considerations are valid in an arbitrary number of dimensions, higher dimensional versions of $\kappa$-Poincaré are in [16].
} 
equations of motion in momentum space for free classical fields have been investigated in [17, 18, 19, 20]. The differential calculus and Dirac equation have been studied in [21, 22]. A field theory has been investigated in [23, 24, 25], and the modification of dispersion relations due to noncommutativity has been related to astrophysical phenomena in [26]. It was argued in [27] that the $\kappa$-Poincaré Hopf algebra that characterizes the symmetries of $\kappa$-Minkowski can be used to introduce the Planck length $(\simeq \lambda)$ directly at the level of the relativity postulates (Doubly Special Relativity).

For the construction of a gauge theory, a *-product is a key tool [28, 29, 30], and * products in the context of $\kappa$-Minkowski generalizing (1.8) have been presented in [23, 24]. On the other side the commutation relations of $\kappa$-Minkowski realize a Lie algebra, and hence the manifold on which they are defined is a Poisson manifold, which has been the subject of intense studies. They culminate with the proof [7] that it is always possible to define a $*$-product on these manifolds, albeit in the space of formal series without warranty of their convergence. In the following we will consider some *-products which define various versions of $\kappa$-Minkowski, and show how they can all be considered generalized Weyl systems. We warn the reader that however our list is not complete. Notably the product defined in [7] will not be considered in this paper. We hope to return to this problem, as well as to the problem of the equivalence of the products, in the future.

\subsection{The $\mathrm{CBH}$ product}

The first product we present is a simple application of the well known Campbell-BakerHausdorff $(\mathrm{CBH})$ formula for the product of the exponential of noncommuting quantities. This $*$-product is actually a particular case of the general product on the cotangent space of a Lie algebra, called the Gutt product [31]. It has also been investigated in the context of deformation quantization in [32, 33, 29, 34, 24]. Let us present this product in a more general way for a set of operators satisfying a Lie algebra condition:

$$
\left[\hat{x}_{\mu}, \hat{x}_{\nu}\right]=c_{\mu \nu}^{\rho} \hat{x}_{\rho}
$$

The CBH formula is:

$$
e^{i \alpha^{\mu} \hat{x}_{\mu}} e^{i \beta^{\nu} \hat{x}_{\nu}}=e^{i \gamma(\alpha, \beta)^{\nu} \hat{x}_{\nu}}
$$

with:

$$
\gamma(\alpha, \beta)^{\mu}=\alpha^{\mu}+\beta^{\mu}+c_{\delta \nu}^{\mu} \alpha^{\delta} \beta^{\nu}+\ldots
$$

The relation(3.5) leads to the associative $\mathrm{CBH}$ star multiplication. The $*$-product defined by this formula is based on a Weyl map with the exponential of the previous expression:

$$
\Omega_{1}(f)=\frac{1}{(2 \pi)^{n / 2}} \int d^{n} k \tilde{f}(k) e^{i k \hat{x}}
$$

Define $*_{1}$ as in 2.12$)$ :

$$
\left(f *_{1} g\right)(x)=\Omega_{1}^{-1}\left(\Omega_{1}(f) \Omega_{1}(g)\right)
$$


Using the CBH formula for the a set of operators $\hat{x}_{\mu}$ satisfying (1.8), the infinite series of nested commutators appearing in the integral can be integrated explicitly [24 to give:

$$
\begin{aligned}
e^{i k^{\mu} \hat{x}_{\mu}} e^{i l^{\nu} \hat{x}_{\nu}} & =e^{i r(k, l)^{\mu} \hat{x}_{\mu}} \\
r^{0} & =k^{0}+l^{0} \\
r^{i} & =\frac{\phi\left(k^{0}\right) e^{\lambda l^{0}} k^{i}+\phi\left(l^{0}\right) l^{i}}{\phi\left(k^{0}+l^{0}\right)} \\
\phi(a) & =\frac{1}{a \lambda}\left(1-e^{-a \lambda}\right)
\end{aligned}
$$

We have that

$$
e^{i k^{\mu} x_{\mu}} *_{1} e^{i l^{\nu} x_{\nu}}=e^{i r(k, l)^{\mu} x_{\mu}}
$$

The product among the generators is obtained differentiating twice (3.12) and setting $k=l=0$ :

$$
\begin{aligned}
x_{0} *_{1} x_{0} & =x_{0}^{2} \\
x_{0} *_{1} x_{i} & =x_{0} x_{i}+\frac{i \lambda}{2} x_{i} \\
x_{i} *_{1} x_{0} & =x_{0} x_{i}-\frac{i \lambda}{2} x_{i} \\
x_{i} *_{1} x_{i} & =x_{i}^{2}
\end{aligned}
$$

of course these reproduce the commutation rules for $\kappa$-Minkowski space time.

\subsection{The Time Ordered Product}

The time ordered product is a modification of the previous one and has its roots in the bicrossproduct structure of $\kappa$-Poincaré. It has been first proposed in [35] and subsequently investigated, for example, in [36, 22, 24]. One defines the time ordering : : for which, in the expansion of a function, all powers of $x_{0}$ appear to the right of the $x_{i}$ 's. The relation for a time ordered exponential is:

$$
e^{i \alpha^{\mu} \hat{x}_{\mu}}=e^{i \phi\left(\alpha_{0}\right) \alpha^{i} \hat{x}_{i}} e^{i \alpha^{0} \hat{x}_{0}}=: e^{i\left(\alpha^{0} \hat{x}_{0}+\phi\left(\alpha_{0}\right) \alpha^{i} \hat{x}_{i}\right)}:
$$

where $\phi$ has been defined in(3.11). The relation(3.14) leads to another associative product via the map

$$
\Omega_{2}(f)=\frac{1}{(2 \pi)^{n / 2}} \int d^{n} k \tilde{f}(k): e^{i k x}:
$$

defined as before by:

$$
\left(f *_{2} g\right)(x)=\Omega_{2}^{-1}\left(\Omega_{2}(f) \Omega_{2}(g)\right)
$$

The product of two exponentials is

$$
e^{i k^{\mu} x_{\mu}} *_{2} e^{i l^{\nu} x_{\nu}}=e^{i\left(k^{0}+l^{0}\right) x_{0}+i\left(k^{i}+e^{\lambda k^{0}} l^{i}\right) x_{i}}
$$


and the product among the generators:

$$
\begin{aligned}
x_{0} *_{2} x_{0} & =x_{0}^{2} \\
x_{0} *_{2} x_{i} & =x_{0} x_{i} \\
x_{i} *_{2} x_{0} & =x_{0} x_{i}-i \lambda x_{i} \\
x_{i} *_{2} x_{i} & =x_{i}^{2}
\end{aligned}
$$

\subsection{The Reduced Moyal Product}

This product is a particular case of a general class of $*$-products for three-dimensional Lie algebras, introduced in [37, which for the $\kappa$-Minkowski case it can be easily generalized to an arbitrary number of dimensions. The idea is to obtain a product in an $n$ dimensional space by considering it as a subspace of an higher dimensional symplectic phase space, equipped with the the usual Poisson bracket, and a Moyal $\star$ product (with deformation parameter $\lambda$ ). The product is then defined by first lifting the functions from the smaller space to the phase space, multiplying them in the higher space, and then projecting back to the smaller space. The form of the lift (a generalized Jordan-Schwinger map) and the canonical structure of the Moyal product ensure that this procedure defines a good * product in the smaller dimensions.

We indicate the coordinate on the phase space $\mathbb{R}^{6}$ with the notation: $\mathbb{R}^{6} \ni u=$ $\left(q_{1}, q_{2}, q_{3} ; p_{1}, p_{2}, p_{3}\right)$. We need to define a map $\pi$ :

$$
\pi: \mathbb{R}^{6} \rightarrow \mathbb{R}^{4}
$$

or equivalently the map $\pi^{*}$ which pulls smooth functions on $\mathbb{R}^{4}$ to smooth functions on $\mathbb{R}^{6}$. The map $\pi$ is explicitly realized with four functions of the $p$ 's and $q$ 's, which we call $x_{\mu}$. The requirement is that the six dimensional Poisson bracket of $x$ 's reproduce the "classical" $\kappa$-Minkowski algebral:

$$
\left\{x_{0}, x_{i}\right\}=x_{i}, \quad\left\{x_{i}, x_{j}\right\}=0 \quad i, j=1,2,3
$$

The $*_{3}$ product is then defined by

$$
\pi^{*}\left(f *_{3} g\right)=\pi^{*} f \star \pi^{*} g
$$

The fact that, after performing the (nonlocal) product in six dimensions, we are left with a function still defined using only the four dimensional coordinates is ensured by the existence of two local functions, $H_{1}$ and $H_{2}$, with the property that

$$
L_{H_{i}}\left(\pi^{*} f\right)=0
$$

\footnotetext{
${ }^{9}$ This algebra is an extension of the three dimensional Lie-algebra $s b(2, \mathbb{C})$ of $2 \times 2$ triangular complex matrices with zero trace treated in 37.
} 
and

$$
L_{H_{i}} \pi^{*}(f(x) \star g(x))=0
$$

In other words, from the six dimensional point of view, the $x$ 's commute with the $H$ 's, and this commutation is stable under the $\star$-product, thus ensuring that if we multiply a function only of the $x$ 's, the product does not depend on the extra coordinates. Upon the identification of the parameter $\theta$ with $\lambda$ we obtain a $\kappa$-Minkowski product on $\mathbb{R}^{4}$.

One of the possible realization of $\pi$ is:

$$
\begin{aligned}
& x_{0}=-\sum_{i} q_{i} p_{i} \\
& x_{i}=q_{i}
\end{aligned}
$$

Two independent commuting functions are:

$$
\begin{aligned}
& H_{1}=\arctan \frac{q_{2}}{q_{3}} \\
& H_{2}=\arctan \frac{q_{3}}{q_{1}}
\end{aligned}
$$

Note however that the representation (3.24) is not unique. For example the following choice works as well:

$$
\begin{aligned}
x_{0}^{\prime} & =-\sum_{i} p_{i} \\
x_{i}^{\prime} & =e^{q_{i}}
\end{aligned}
$$

with the commuting functions

$$
\begin{aligned}
& H_{1}^{\prime}=e^{q_{2}-q_{3}} \\
& H_{2}^{\prime}=e^{q_{3}-q_{1}}
\end{aligned}
$$

connected by a singular canonical transformation to the previous one.

We will denote the products with the choices (3.24) and (3.26) as $*_{3}$ and $*_{4}$ respectively. The explicit products between the generators are in [37]:

$$
\begin{aligned}
x_{0} *_{3} x_{0} & =x_{0}^{2}+\frac{3}{4} \lambda^{2} \\
x_{0} *_{3} x_{i} & =x_{0} x_{i}+i \frac{\lambda}{2} x_{i} \\
x_{i} *_{3} x_{0} & =x_{0} x_{i}-i \frac{\lambda}{2} x_{i} \\
x_{i} *_{3} x_{i} & =x_{i}^{2}
\end{aligned}
$$

and

$$
x_{0} *_{4} x_{0}=x_{0}^{2}
$$




$$
\begin{aligned}
x_{0} *_{4} x_{i} & =x_{0} x_{i}+i \frac{\lambda}{2} x_{i} \\
x_{i} *_{4} x_{0} & =x_{0} x_{i}-i \frac{\lambda}{2} x_{i} \\
x_{i} *_{4} x_{i} & =x_{i}^{2}
\end{aligned}
$$

It may be noticed that these relations are the same as the one for $*_{1}$ in (3.13). The two products however, although similar, do not coincide for generic functions.

\section{$4 \quad$ Weyl Systems for $\kappa$-Minkowski}

In this section we will show how the various products presented earlier are particular instances of generalized Weyl systems. The starting point for the construction of the product is the identification of the $W(k)$ 's, which enables the calculation of the particular relation (2.17) for the various cases. In other words we will give an explicit realization of the group composition law $\oplus$. All Weyl systems presented here have $\omega=0$, and in all cases a straightforward calculation verifies relation (2.40).

\subsection{Weyl System for the $\mathrm{CBH}$ product}

In this case the relation is given by the $\mathrm{CBH}$ product, which for $\kappa$-Minkowski has been given in eqs. (3.5.3.11). The composition $\oplus_{1}$ can be calculated to give:

$$
\begin{aligned}
\left(k \oplus_{1} k^{\prime}\right)^{0} & =k^{0}+k^{\prime 0} \\
\left(k \oplus_{1} k^{\prime}\right)^{i} & =\frac{\phi\left(k^{0}\right) k^{i}+e^{\lambda k^{0}} \phi\left({k^{\prime}}^{0}\right) k^{\prime i}}{\phi\left(k^{0}+k^{\prime 0}\right)}
\end{aligned}
$$

where $\phi$ has been defined in eq. (3.11). We have that

$$
e^{i k \hat{x}}=\Omega\left(e^{i k x}\right)
$$

There is one check which has to be performed to ensure that $\oplus_{1}$ defines a group. Relation (2.38) is verified with the definition

$$
\bar{k}=\left(-k^{0},-k^{i}\right)
$$

in fact

$$
\overline{\left(k \oplus_{1} k^{\prime}\right)}=\overline{k^{\prime} \oplus \bar{k}}
$$

\subsection{Weyl System for the Time Ordered Product}

This case is similar to the previous one, and a direct calculation using the CBH relations for the time ordered exponentials give:

$$
\begin{aligned}
& \left(k \oplus_{2} k^{\prime}\right)^{0}=k^{0}+{k^{\prime}}^{0} \\
& \left(k \oplus_{2} k^{\prime}\right)^{i}=k^{i}+e^{\lambda k^{0}} k^{\prime i}
\end{aligned}
$$


with

$$
\bar{k}=\left(-k^{0},-e^{-\lambda k^{0}} k^{i}\right)
$$

This momenta composition reflects the coproduct in the bicrossproduct basis, thus rendering the time ordered exponential a natural basis for the space of functions in this case. Note that

$$
: e^{i k \hat{x}}:=\Omega_{1}\left(e^{i k^{i} x_{i}} *_{1} e^{i k^{0} x_{0}}\right)
$$

So the product $*_{2}$ can be expressed by the choice of a different $W$ of the $*_{1}$ product.

\subsection{Weyl Systems for the Reduced Moyal products}

The path to the definition of the reduced products is intrinsically different from the first two. These are defined using straightforwardly the $\mathrm{CBH}$ formula with a specific ordering. The reduced product instead comes from a four dimensional reduction of a six dimensional product. There is therefore no warranty that it is possible to obtain them form a four dimensional Weyl system. This is nevertheless possible.

Notice that if we were to define $W$ as the $*_{3}$ or $*_{4}$ exponential of $i k^{\mu} x_{\mu}$ we would find the $\mathrm{CBH}$ product. This is because the $*$ commutator of the $x$ 's are all the same. We must therefore use another quantity, and we could use the ordinary exponential. Care must be taken however because this is not an unitary operator (with the hermitean conjugation defined in eq. (2.31)). So that it is necessary to normalize it. The calculations of the product of two exponentials are in Appendices $\mathrm{A}$ and $\mathrm{B}$. We define $W_{3}$ as

$$
W_{3}(k)=\left|a\left(k^{0},-k^{0}\right)\right|^{3 / 2} \Omega_{3}\left(e^{i k x}\right)
$$

with

$$
\begin{aligned}
a\left(k^{0}, l^{0}\right) & =1+\frac{\lambda^{2}}{4} k^{0} l^{0} \\
b\left(k^{0}\right) & =1+\frac{\lambda}{2} k^{0}
\end{aligned}
$$

From equation (A.15) we can read

$$
\left(k \oplus_{3} k^{\prime}\right)^{\mu}=k^{\mu}+k^{\mu \prime}-\frac{\lambda}{2 a}\left(k^{\prime 0} b\left(k^{0}\right) k^{\mu}-k^{\prime 0} b\left(-k^{\prime 0}\right) k^{\prime \mu}\right)
$$

with $\bar{k}=-k$. Unlike all other composition laws, $\oplus_{3}$ is the only one in which the "timelike" coordinate has a nonabelian structure: $\operatorname{Tr}\left(k \oplus_{3} k^{\prime}\right)^{0} \neq\left(k^{\prime} \oplus_{3} k\right)^{0}$.

For the $*_{4}$ case, the exponential is unitary, and it is possible to define $W_{4}(k)=e^{i k x}$. In Appendix B we calculate the composition rule $\oplus_{4}$ which results:

$$
k \oplus_{4} k^{\prime}=\left(k^{0}+{k^{\prime}}^{0}, e^{-\frac{\lambda}{2} k^{\prime}} \vec{k}+e^{\frac{\lambda}{2} k^{0}} \vec{k}^{\prime}\right)
$$

\footnotetext{
${ }^{10}$ Note also that $\oplus_{3}$ is not well defined for all $\left(k, k^{\prime}\right)$, so that we do not have a group. The integral (2.29) is anyway well defined.
} 
with $\bar{k}=-k$.

Notice that, while the composition law $\oplus_{2}$ is connected to the coproduct (3.3) in the bicrossproduct basis, the $\oplus_{4}$ is related to the coproduct in the standard basis [9]:

$$
\begin{aligned}
\Delta P_{0} & =P_{0} \otimes 1+1 \otimes P_{0} \\
\Delta M_{i} & =M_{i} \otimes 1+1 \otimes M_{i} \\
\Delta P_{i} & =P_{i} \otimes e^{-\frac{\lambda P_{0}}{2}}+P_{i} \otimes e^{\frac{\lambda P_{0}}{2}} \\
\Delta N_{i} & =N_{i} \otimes e^{\frac{\lambda P_{0}}{2}}+e^{-\frac{\lambda P_{0}}{2}} \otimes N_{i}+\frac{\lambda}{2} \varepsilon_{i j k}\left(P_{j} \otimes M_{k} e^{\frac{\lambda P_{0}}{2}}+e^{-\frac{\lambda P_{0}}{2}} M_{j} \otimes P_{k}\right)
\end{aligned}
$$

and in particular to the symmetric coproduct for $P_{i}$. The similarity goes beyond it, as the $*_{4}$ product can be written as a symmetrically ordered" product with respect to the $x_{0}$ coordinate defining:

$$
\ddagger e^{i k \hat{x}} \ddagger=e^{\frac{i k^{0}}{2} \hat{x}_{0}} e^{-i k^{i} \hat{x}_{i}} e^{\frac{i k^{0}}{2} \hat{x}_{0}}
$$

so that

$$
\ddagger e^{i k \hat{x}} \ddagger \ddagger e^{i l \hat{x}} \ddagger=\ddagger e^{i\left(k \oplus_{4} l\right) \hat{x}} \ddagger
$$

In analogy with (4.7) we have

$$
W_{4}(k)=\Omega_{1}\left(e^{\frac{i k^{0}}{2} x_{0}} *_{1} e^{-i k^{i} x_{i}} *_{1} e^{\frac{i k^{0}}{2} x_{0}}\right)
$$

\section{Conclusions}

A noncommutative geometry is defined by a *-algebra, and deformations of the algebras of functions on a manifold are particularly important examples. In this paper we have shown that it is in principle possible to obtain noncommutative products between functions which generalize the commutation relations of Lie algebras, possibly with central extensions. This has been done generalizing Weyl systems by the substitution of the usual abelian sum with a nonabelian composition law.

We have seen that four different $*$-products which generalize the commutation relations of $\kappa$-Minkowski fit nicely in this framework. This enabled us to perform a comparative study of these products, and to discover several relations among them, and with the coproducts and other structures which characterize the quantum algebra $\kappa$-Poincaré. These consideration will be helpful for the ultimate goal of the construction of field theories on these noncommutative spaces.

\section{Acknowledgments}

We thank G. Amelino-Camelia, J.M. Gracia-Bondía, G. Marmo and J. C. Várilly for helpful discussions and correspondence. The work of F.L. is supported in part by the Progetto di Ricerca di Interesse Nazionale SInteSi 2000. 


\section{A Calculation of the $*_{3}$ product of two exponentials}

In this appendix we give some details of the calculations of the product of two (ordinary) exponential functions. We will use the following (equivalent) form of the product (1.3) for the product of two functions $f, g$ on $\mathbb{R}^{6}$ :

$$
f(u) \star g(u)=(2 \pi)^{-6} \int d^{6} s d^{6} t f\left(u+\frac{\lambda}{2} J_{6} s\right) g(u+t) e^{-i s t}
$$

where $u=\left(q_{1}, \ldots p_{3}\right)$ and $J$ denotes the antisymmetric matrix:

$$
J_{6}=\left(\begin{array}{cc}
0 & I_{3} \\
-I_{3} & 0
\end{array}\right)
$$

Let us express $u=(\vec{q}, \vec{p})$ and introduce the following notation:

$$
\begin{aligned}
u & =(\vec{q}, \vec{p}) \\
s & =\left(\vec{s}_{1}, \vec{s}_{2}\right) \\
t & =\left(\overrightarrow{t_{1}}, \overrightarrow{t_{2}}\right)
\end{aligned}
$$

in which all vectors belong to $\mathbb{R}_{3}$. Using the integral form for a Moyal product (A.1), the deformed (six dimensional) product of two exponential is:

$$
e^{i k^{\mu} x_{\mu}} \star e^{i l^{\mu} x_{\mu}}=(2 \pi)^{-6} \int d^{6} s d^{6} t e^{i k^{\mu} x_{\mu}\left(u+\frac{\lambda}{2} J_{6} s\right)} e^{i l^{\mu} x_{\mu}(u+t)} e^{-i s t}
$$

with (using (3.24) for the last step):

$$
i k^{\mu} x_{\mu}(u) \equiv i k x=i k^{0} x_{0}-i \vec{k} \cdot \vec{x}=-i\left(k^{0} \vec{q} \cdot \vec{p}+\vec{k} \cdot \vec{q}\right)
$$

the arguments of the $x$ 's become

$$
\begin{gathered}
u+\frac{\lambda}{2} J_{6} s=\left(\vec{q}+\frac{\lambda}{2} \overrightarrow{s_{2}}, \vec{p}-\frac{\lambda}{2} \overrightarrow{s_{1}}\right) \\
i k^{\mu} x_{\mu}\left(u+\frac{\lambda}{2} J_{6} s\right)=-i\left[k^{0}\left(\vec{q}+\frac{\lambda}{2} \overrightarrow{s_{2}}\right)\left(\vec{p}-\frac{\lambda}{2} \overrightarrow{s_{1}}\right)+\vec{k} \cdot\left(\vec{q}+\frac{\lambda}{2} \overrightarrow{s_{2}}\right)\right] \\
=-i\left(k^{0} \vec{q} \cdot \vec{p}+\vec{k} \cdot \vec{q}\right)-i \frac{\lambda}{2}\left(k^{0}\left(\overrightarrow{s_{2}} \cdot \vec{p}-\vec{q} \cdot \overrightarrow{s_{1}}\right)+\vec{k} \cdot \vec{s}_{2}\right)+i \frac{\lambda^{2}}{4} k^{0} \vec{s}_{1} \cdot \vec{s}_{2} \\
=i k x-i \frac{\lambda}{2}\left(k^{0}\left(\overrightarrow{s_{2}} \cdot \vec{p}-\vec{q} \cdot \overrightarrow{s_{1}}\right)+\vec{k} \cdot \overrightarrow{s_{2}}\right)+i \frac{\lambda^{2}}{4} k^{0} \vec{s}_{1} \cdot \vec{s}_{2} \\
i l^{\mu} x^{\mu}(u+t)=-i\left[l^{0}\left(\vec{q}+\overrightarrow{t_{1}}\right)\left(\vec{p}+\overrightarrow{t_{2}}\right)+\vec{l} \cdot\left(\vec{q}+\overrightarrow{t_{1}}\right)\right] \\
=i l x-i\left(l^{0}\left(\vec{q} \cdot \overrightarrow{t_{2}}+\vec{p} \cdot \overrightarrow{t_{1}}+\overrightarrow{t_{1}} \cdot \overrightarrow{t_{2}}\right)+\vec{l} \cdot \overrightarrow{t_{1}}\right)
\end{gathered}
$$

and

$$
\begin{aligned}
e^{i k^{\mu} x_{\mu}} \star & e^{i l^{\mu} x_{\mu}}=(2 \pi)^{-6} e^{i(k+l) x} \int d \vec{s}_{1} d \vec{s}_{2} d \vec{t}_{1} d \vec{t}_{2} e^{-i \frac{\lambda}{2}\left(k^{0}\left(\vec{s}_{2} \cdot \vec{p}-\vec{q} \cdot \vec{s}_{1}\right)+\vec{k} \cdot \vec{s}_{2}\right)+i \frac{\lambda^{2}}{4} k^{0} \vec{s}_{1} \cdot \vec{s}_{2}} \\
& e^{-i\left(l^{0}\left(\vec{q} \cdot \vec{t}_{2}+\vec{p} \cdot \vec{t}_{1}+\vec{t}_{1} \cdot \vec{t}_{2}\right)+\vec{l} \cdot \vec{t}_{1}\right)} e^{-i\left(\vec{s}_{1} \cdot \vec{t}_{1}+\vec{s}_{2} \cdot \vec{t}_{2}\right)}
\end{aligned}
$$


Reordering the exponentials:

$$
\begin{aligned}
e^{i k^{\mu} x_{\mu}} \star e^{i l^{\mu} x_{\mu}=} & (2 \pi)^{-6} e^{i(k+l) x} \int d \vec{s}_{1} d \vec{s}_{2} d \overrightarrow{t_{1}} d \vec{t}_{2} e^{i \vec{s}_{1}\left(\frac{\lambda}{2} k^{0} \vec{q}+\frac{\lambda^{2}}{4} k^{0} \vec{s}_{2}-\vec{t}_{1}\right)} \\
& e^{-i \vec{t}_{2}\left(l^{0} \vec{t}_{1}+l^{0} \vec{q}+\vec{s}_{2}\right)} e^{-i \frac{\lambda}{2} \vec{s}_{2}\left(k^{0} \vec{p}+\vec{k}\right)-i \vec{t}_{1} \cdot\left(\vec{l}+l^{0} \vec{p}\right)}
\end{aligned}
$$

at this point we can make the integration in the $\vec{s}_{1}$ e $\overrightarrow{t_{2}}$ variables:

$$
\begin{aligned}
\frac{1}{(2 \pi)^{3}} \int d \vec{s}_{1} e^{i \vec{s}_{1}\left(\frac{\lambda}{2} k^{0} \vec{q}+\frac{\lambda^{2}}{4} k^{0} \vec{s}_{2}-\vec{t}_{1}\right)} & =\delta^{(3)}\left(\frac{\lambda}{2} k^{0} \vec{q}+\frac{\lambda^{2}}{4} k^{0} \vec{s}_{2}-\vec{t}_{1}\right) \\
\frac{1}{(2 \pi)^{3}} \int d \vec{t}_{2} e^{-i \vec{t}_{2}\left(l^{0} \vec{q}+l^{0} \vec{t}_{1}+\vec{s}_{2}\right)} & =\delta^{(3)}\left(l^{0} \vec{q}+l^{0} \vec{t}_{1}+\vec{s}_{2}\right)
\end{aligned}
$$

to obtain:

$$
\begin{aligned}
e^{i k^{\mu} x_{\mu}} \star e^{i l^{\mu} x_{\mu}}= & e^{i(k+l) x} \int d \vec{s}_{2} d \vec{t}_{1} \delta^{(3)}\left(\frac{\lambda}{2} k^{0} \vec{q}+\frac{\lambda^{2}}{4} k^{0} \vec{s}_{2}-\vec{t}_{1}\right) \delta^{(3)}\left(l^{0} \vec{q}+l^{0} \vec{t}_{1}+\vec{s}_{2}\right) \\
& e^{-i \frac{\lambda}{2} \vec{s}_{2}\left(k^{0} \vec{p}+\vec{k}\right)-i \vec{t}_{1} \cdot\left(\vec{l}+l^{0} \vec{p}\right)}
\end{aligned}
$$

and making integral in $d \vec{t}_{1}$ we have:

$$
\begin{aligned}
e^{i k^{\mu} x_{\mu}} \star e^{i l^{\mu} x_{\mu}=} & e^{i(k+l) x} \int d \vec{s}_{2} e^{-i \frac{\lambda}{2} \vec{s}_{2} \cdot\left(k^{0} \vec{p}+\vec{k}\right)-i \frac{\lambda^{2}}{4} k^{0} \vec{s}_{2}\left(\vec{l}+l^{0} \vec{p}\right)-i \frac{\lambda}{2} k^{0} \vec{q}\left(\vec{l}+l^{0} \vec{p}\right)} \\
& \delta^{(3)}\left(\vec{s}_{2}\left(1+\frac{\lambda^{2}}{4} k^{0} l^{0}\right)+l^{0}\left(1+\frac{\lambda}{2} k^{0}\right) \vec{q}\right)
\end{aligned}
$$

Using $a$ and $b$ defined in (4.9), we make the last integration to obtain:

$$
\delta\left(\vec{s}_{2} a\left(k^{0}, l^{0}\right)+l^{0} b\left(k^{0}\right) \vec{q}\right)=\frac{1}{\left|a\left(k^{0}, l^{0}\right)\right|^{3}} \delta\left(\vec{s}_{2}+l^{0} \frac{b\left(k^{0}\right)}{a\left(k^{0}, l^{0}\right)} \vec{q}\right)
$$

which fixes $\vec{s}_{2}=-l^{0} \frac{b}{a} \vec{q}$, so that the integral becomes:

$$
\begin{aligned}
& \frac{1}{\left|a\left(k^{0}, l^{0}\right)\right|^{3}} e^{i \frac{\lambda}{2} l^{0} \frac{b}{a} \vec{q} \cdot\left(k^{0} \vec{p}+\vec{k}\right)+i \frac{\lambda^{2}}{4} k^{0} l^{0} \frac{b}{a} \vec{q}\left(\vec{l}+l^{0} \vec{p}\right)-i \frac{\lambda}{2} k^{0} \vec{q}\left(\vec{l}+l^{0} \vec{p}\right)} \\
= & \frac{1}{\left|a\left(k^{0}, l^{0}\right)\right|^{3}} e^{i \frac{\lambda}{2} l^{0} \frac{b}{a}\left(-k^{0} x_{0}+\vec{k} \cdot \vec{x}\right)+i \frac{\lambda^{2}}{4} k^{0} l^{0} \frac{b}{a}\left(\vec{l} \cdot \vec{x}-l^{0} x_{0}\right)-i \frac{\lambda}{2} k^{0}\left(\vec{l} \cdot \vec{x}-l^{0} x_{0}\right)} \\
= & \frac{1}{\left|a\left(k^{0}, l^{0}\right)\right|^{3}} e^{-i \frac{\lambda}{2} l^{0} \frac{b}{a} k x-i \frac{\lambda^{2}}{4} k^{0} l^{0} \frac{b}{a} l x+i \frac{\lambda}{2} k^{0} l x} \\
= & \frac{1}{\left|a\left(k^{0}, l^{0}\right)\right|^{3}} e^{-i \frac{\lambda}{2 a}\left(l^{0} b k+\frac{\lambda}{2} k^{0} l^{0} b l-\frac{\lambda}{2} k^{0} l a\right) x} \\
= & \frac{1}{\left|a\left(k^{0}, l^{0}\right)\right|^{3}} e^{-i \frac{\lambda}{2 a}\left(l^{0} b\left(k^{0}\right) k-k^{0} b\left(-l^{0}\right) l\right) x}
\end{aligned}
$$

The final result is:

$$
e^{i k x} \star e^{i l x}=\frac{1}{\left|a\left(k^{0}, l^{0}\right)\right|^{3}} e^{i(k+l) x} e^{-i \frac{\lambda}{2 a\left(k^{0}, l^{0}\right)}\left(l^{0} b\left(k^{0}\right) k-k^{0} b\left(-l^{0}\right) l\right) x}=\frac{1}{\left|a\left(k^{0}, l^{0}\right)\right|^{3}} e^{i\left(k \oplus_{3} l\right) x}
$$

It results also $\bar{k}=-k$. From relation (A.15) can be seen that the function $e^{i k x}$ is not unitary for the product $*_{3}$, and to make it unitary one should renormalize it dividing by $\left|a\left(k^{0}, k^{0}\right)\right|^{3 / 2}$, thus finding (4.8). 


\section{B Calculation of the $*_{4}$ product of two exponentials}

In this second appendix we consider the map given by relation (3.26), which gives rise to the product $*_{4}$. Again with the use of (A.1) we calculate the product among exponential functions:

$$
f(u)=e^{i k x}, \quad g(u)=e^{i l x}
$$

where $x=x(u)$ and $u, s, t$ are defined as in the previous appendix. We have then:

$$
\begin{aligned}
i k x\left(u+\frac{1}{2} \lambda J s\right) & =-i\left(k^{0} \sum_{i=1}^{3}\left(p_{i}-\frac{\lambda}{2} s_{1 i}\right)+\sum_{i=1}^{3} k_{i} e^{q_{i}+\frac{\lambda}{2} s_{2}}\right) \\
& =k^{0} x_{0}+i \sum_{i=1}^{3}\left(\frac{\lambda}{2} k^{0} s_{1 i}-k_{i} x_{i} e^{\frac{\lambda}{2} s_{2 i}}\right) \\
i l x(u+t) & =-i \sum_{i=1}^{3}\left(l^{0}\left(p_{i}+t_{2 i}\right)+l_{i} e^{q_{i}+t_{1 i}}\right) \\
& =l^{0} x_{0}-i \sum_{i=1}^{3}\left[l^{0} t_{2 i}+l_{i} x_{i} e^{t_{1 i}}\right]
\end{aligned}
$$

Performing the integral:

$$
\begin{aligned}
e^{i k x} *_{4} e^{i l x} & =e^{i\left(k^{0}+l^{0}\right) x_{0}} \int d s d t e^{i \sum_{i}\left(\frac{\lambda}{2} k^{0} s_{1 i}-k_{i} x_{i} e^{\frac{\lambda}{2} s_{2 i}}\right)} e^{i \sum_{i}^{3}\left(-l^{0} t_{2 i}-l_{i} x_{i} e^{t_{1 i}}\right)} e^{-i \sum_{i}^{3}\left(s_{1 i} t_{1 i}+s_{2 i} t_{2 i}\right)} \\
& =e^{i\left(k^{0}+l^{0}\right) x_{0}} \prod_{i}^{3} \int d s_{2_{i}} d t_{1 i} \delta\left(\frac{\lambda}{2} k^{0}-t_{1 i}\right) \delta\left(l^{0}+s_{2 i}\right) e^{-i k_{i} x_{i} e^{\frac{\lambda}{2} s_{2 i}}} e^{-i l_{i} x_{i} e^{t_{1 i}}} \\
& =e^{i\left(k^{0}+l^{0}\right) x_{0}} \prod_{i}^{3}\left[e^{-i k_{i} x_{i} e^{-\frac{\lambda}{2} l^{0}}} e^{-i l_{i} x_{i} e^{\frac{\lambda}{2} k^{0}}}\right] \\
& =e^{i\left(k^{0}+l^{0}\right) x_{0}}\left[e^{-i \vec{k} \vec{x} e^{-\frac{\lambda}{2} l^{0}}} e^{-i \overrightarrow{\vec{x}} e^{\frac{\lambda}{2} k^{0}}}\right] \\
& =e^{i(k \oplus 4) x}
\end{aligned}
$$

\section{References}

[1] A. Connes, Noncommutative Geometry, Academic Press, (1994); G. Landi, An introduction to noncommutative spaces and their geometry, Springer (1998), arXiv:hepth/9701078. J. Madore, An Introduction to Noncommutative Differential Geometry and Physical Applications, LMS 257, Cambridge Univerisity Press (1998).

[2] J. M. Gracia-Bondía, J. C. Várilly and H. Figueroa, Elements of Noncommutative Geometry, Birkhäuser, (2001).

[3] H. S. Snyder, "Quantized Space-Time," Phys. Rev. 71 (1947) 38. 
[4] S. Doplicher, K. Fredenhagen and J. E. Roberts, "The Quantum Structure of SpaceTime at the Planck Scale and Quantum Fields," Commun. Math. Phys. 172 (1995) 187; "Space-time quantization induced by classical gravity," Phys. Lett. B 331 (1994) 39.

[5] H.J. Grönewold, "On the Principles of Elementary Quantum Mechanics", Physica 12 (1946) 405; J.E. Moyal, "Quantum Mechanics as a Statistical Theory", Proc. Cambridge Phil. Soc. 45 (1949) 99.

[6] F. Bayen, M. Flato, C. Fronsdal, A. Lichnerowicz and D. Sternheimer, "Deformation theory and quantization. I. Deformation of symplectic structures", Ann. Phys. (NY) 111 (1978) 61; "Deformation theory and quantization. II. Physical applications", Ann. Phys. (NY) 111 (1978) 111.

[7] M. Kontsevich, "Deformation quantization of Poisson manifolds I", g-alg/9709040.

[8] S. Zakrzewski, "Quantum Poincaré group related to the $\kappa$-Poincaré algebra", J. of Phys. A27 (1994) 2075.

[9] J. Lukierski, H. Ruegg, A. Nowicki and V. N. Tolstoi, "Q-deformation of Poincaré algebra," Phys. Lett. B 264 (1991) 331; J. Lukierski, A. Nowicki and H. Ruegg, "New quantum Poincaré algebra and k deformed field theory," Phys. Lett. B 293 (1992) 344.

[10] A. Ballesteros, F. J. Herranz, M. A. del Olmo and M. Santander, "4-D quantum affine algebras and space-time q symmetries," J. Math. Phys. 35 (1994) 4928 arXiv:hepth/9310140]; “A New 'Null Plane' Quantum Poincaré Algebra," Phys. Lett. B 351 (1995) 137.

[11] E. Celeghini, R. Giachetti, E. Sorace and M. Tarlini, "Three Dimensional Quantum Groups from Contraction of $S U(2)_{Q}$," J. Math. Phys. 31 (1990) 2548.

[12] S. Majid and H. Ruegg, "Bicrossproduct structure of kappa Poincaré group and noncommutative geometry," Phys. Lett. B 334 (1994) 348 arXiv:hep-th/9405107.

[13] H. Weyl, The theory of groups and Quantum Mechanics, Dover, 1931

[14] J. Baez and I.E.Segal and Z. Zhou, Introduction to Algebraic and constructive Quantum Field Theory, Princeton University Press (1992).

[15] J. M. Gracia-Bondía and J. C. Várilly, "Algebras of distributions suitable for phasespace quantum mechanics. I", J. Math. Phys. 29 (1988) 869.

[16] J. Lukierski and H. Ruegg, "Quantum Kappa Poincaré in any Dimension," Phys. Lett. B 329 (1994) 189 arXiv:hep-th/9310117.

[17] J. Lukierski, H. Ruegg and W. Ruhl, "From Kappa Poincaré Algebra to Kappa Lorentz Quasigroup: a Deformation of Relativistic Symmetry," Phys. Lett. B 313 (1993) 357.

[18] S. Giller, C. Gonera, P. Kosinski, M. Majewski, P. Maslanka and J. Kunz, "On Q-Covariant Wave Functions," Mod. Phys. Lett. A 8 (1993) 3785.

[19] P. Maslanka, "Deformation map and Hermitian representations of k-Poincaré algebra", J. Math. Phys 34 (1993) 6025.

[20] A. Nowicki, E. Sorace and M. Tarlini, "The Quantum deformed Dirac equation from the kappa Poincaré algebra," Phys. Lett. B 302 (1993) 419 arXiv:hep-th/9212065. 
[21] P. N. Bibikov, "Dirac operator on kappa-Minkowski space, bicovariant differential calculus and deformed U(1) gauge theory," J. Phys. A 31 (1998) 6437 arXiv:qalg/9710019].

[22] P. Kosinski, P. Maslanka, J. Lukierski and A. Sitarz, "Towards Kappa-Deformed D = 4 Relativistic Field Theory," Czech. J. Phys. 48 (1998) 1407.

[23] P. Kosinski, J. Lukierski and P. Maslanka, "Local D $=4$ field theory on kappadeformed Minkowski space," Phys. Rev. D 62 (2000) 025004 arXiv:hep-th/9902037;

[24] P. Kosinski, J. Lukierski and P. Maslanka, "kappa-deformed Wigner construction of relativistic wave functions and free fields on kappa-Minkowski space," Nucl. Phys. Proc. Suppl. 102 (2001) 161 arXiv:hep-th/0103127.

[25] G. Amelino-Camelia and M. Arzano, "Coproduct and star product in field theories on Lie-algebra non-commutative space-times," Phys. Rev. D 65 (2002) 084044 arXiv:hep-th/0105120.

[26] G. Amelino-Camelia and S. Majid, "Waves on noncommutative spacetime and gamma-ray bursts," Int. J. Mod. Phys. A 15 (2000) 4301 arXiv:hep-th/9907110.

[27] G. Amelino-Camelia, "Relativity in space-times with short-distance structure governed by an observer-independent (Planckian) length scale," Int. J. Mod. Phys. D 11 (2002) 35 arXiv:gr-qc/0012051; G. Amelino-Camelia, "Testable scenario for relativity with minimum-length," Phys. Lett. B 510 (2001) 255 arXiv:hep-th/0012238.

[28] N. Seiberg and E. Witten, "String theory and noncommutative geometry," JHEP 9909 (1999) 032 arXiv:hep-th/9908142.

[29] J. Madore, S. Schraml, P. Schupp and J. Wess, "Gauge theory on noncommutative spaces," Eur. Phys. J. C 16 (2000) 161 arXiv:hep-th/0001203.

[30] R. J. Szabo, "Quantum field theory on noncommutative spaces," arXiv:hepth/0109162.

[31] S. Gutt, "An Explicit *-product on the Cotangent Bundle of a Lie Group", Lett. Math. Phys. 7 (1983) 249.

[32] V. Kathotia, "Kontsevich's Universal Formula for Deformation Quantization and the Campbell-Baker-Hausdorff Formula, I", arXiv:math.QA/9811174.

[33] B. Shoikhet, "On the Kontsevich and the Campbell-Baker-Hausdorff deformation quantizations of a linear Poisson structure", arXiv:math.QA/9903036.

[34] B. Jurco, S. Schraml, P. Schupp and J. Wess, "Enveloping algebra valued gauge transformations for non-Abelian gauge groups on non-commutative spaces," Eur. Phys. J. C 17 (2000) 521 arXiv:hep-th/0006246.

[35] S. Majid, "Quasitriangular Hopf Algebras And Yang-Baxter Equations," Int. J. Mod. Phys. A 5 (1990) 1.

[36] S. Majid and R. Oeckl, "Twisting of quantum differentials and the Planck scale Hopf algebra," Commun. Math. Phys. 205 (1999) 617 arXiv:math.qa/9811054.

[37] J. M. Gracia-Bondia, F. Lizzi, G. Marmo and P. Vitale, "Infinitely many star products to play with," JHEP 0204 (2002) 026 arXiv:hep-th/0112092. 